\title{
Genetic Structure Analysis of the Alien Mollusk Stenomphalia ravergiensis (Mollusca, Gastropoda, Pulmonata) Population in Belgorod (Russia)
}

\author{
E. A. Snegin* and V. V. Adamova \\ Belgorod National Research University, Belgorod, 308015 Russia \\ *e-mail: snegin@bsu.edu.ru \\ Received November 15, 2016
}

\begin{abstract}
Gel electrophoretic analysis of allozymes was used to study the genetic structure of the population of the alien mollusk Stenomphalia ravergiensis that inhabits urbanized landscapes in the town of Belgorod (Russia). A high genetic variability level and low values of inbreeding coefficient were characteristic of most colonies. Weak genetic separation of the snail groups studied was also demonstrated. Invasive colony distribution was supposed to correspond to the island model. The calculation of effective population size and the comparison of this value to the respective parameters of native and invasive species of terrestrial mollusks revealed a high viability level for the population of the species of interest in the study area.
\end{abstract}

Keywords: alien species, terrestrial mollusk, genetic structure, allozymes

DOI: $10.1134 /$ S2075111717040117

\section{INTRODUCTION}

Inadvertent introduction of plants and animals to various habitats on the planet is known to have unpredictable consequences sometimes, both for introduced species themselves and for the aboriginal community members. At the same time, invasive colonies exposed to novel living conditions can serve as good natural models for the validation of our concepts concerning the evolutionary mechanisms. As conventionally believed, the founder effect is manifested in such colonies (Mayr, 1954), and the combination of this effect and further inbreeding leads to allelic diversity reduction and ultimately to destabilization and extinction. This is one of the possible reasons for the unsuccessful outcomes of introduction in certain cases. Therefore, the expansion of a species beyond the native range implies a rather high level of genetic and phenotypic variability, which allows for adaptation to novel conditions and new habitat colonization (Alimov and Bogutskaya, 2004; Yeh and Trevor, 2004; Ghalambor et al., 2007; Lee and Gelembiuk, 2008). Moreover, compensatory mechanisms that alleviate the negative effect of inbreeding must be active in the genomes of such species (Snegin, 2012).

A small colony of the terrestrial mollusk Stenomphalia ravergiensis (Férussac, 1835) was found in the town of Belgorod in 2002 (Snegin and Prisnyi, 2008). The historical range of this species is located in the Northern Caucasus and Transcaucasia, mostly in the forest belt (Shileiko, 1978), and sometimes in open areas and on crystal- line highland massifs (Akramovskii, 1976; Kiyashko, 2007). The species was detected in different parts of Belgorod and in the environs of the town in the following years. Notably, active expansion of this species beyond the northern border of the range in Eastern Europe was observed in recent decades (Snegin and Prisnyi, 2008; Balashov et al., 2013). This type of invasive process development can possibly have a pronounced effect on the local malacofauna and the local ecosystems as a whole (Clarke et al., 1984; Murray et al., 1988; Parker et al., 1999; Coote and Loeve, 2003).

The aim of the present work was to analyze the genetic structure of invasive $S$. ravergiensis colonies using allozyme markers in order to assess the viability level of the mollusks and forecast the character of the invasive process.

\section{MATERIALS AND METHODS}

The present study continues our research on demographic and conchiometric parameters of $S$. ravergiensis colonies in Belgorod (Snegin and Adamova, 2016). Individuals from seven isolated $S$. ravergiensis groups (Fig. 1, Table 1) were collected in the town of Belgorod and its environs during the summer field season of 2015. Twenty-five sexually mature individuals were collected at each site. A sample of 68 individuals of the same species collected from a population that inhabited the native range (Noratus, Armenia) was used for comparison. 


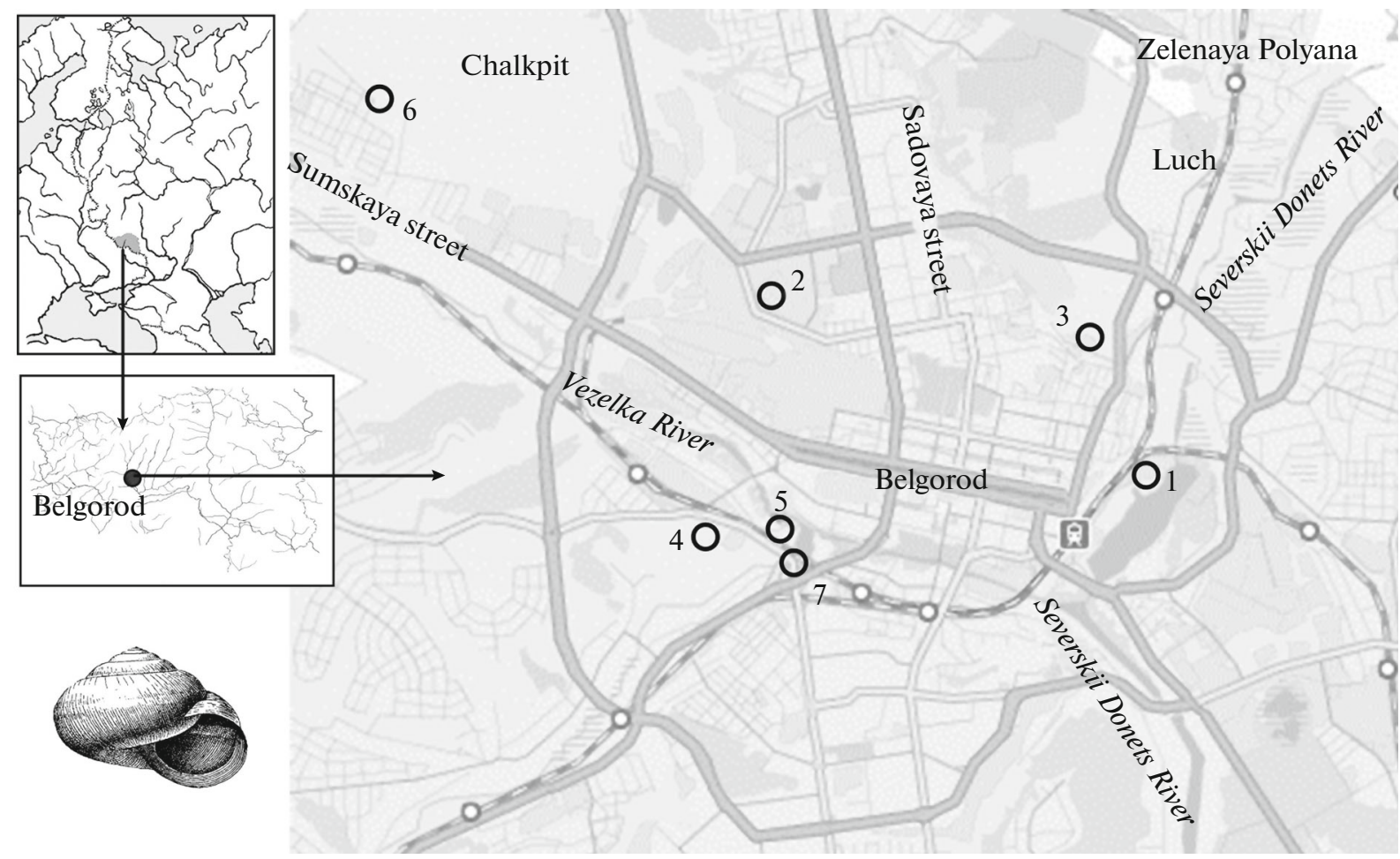

Fig. 1. Stenomphalia ravergiensis sampling sites: (1) Donets, (2) Michurina, (3) Kalinina, (4) Botanicheskii sad, (5) Vezelka, (6) Kar'er, (7) Vodstroi.

Isoenzymes were extracted from the mollusk foot retractor by freezing the tissue at $-80^{\circ} \mathrm{C}$ with subsequent thawing and mechanical homogenization by a Teflon pestle in $0.05 \mathrm{M}$ Tris- $\mathrm{HCl}$ buffer ( $\mathrm{pH}$ 6.7). Polyacrylamide gel electrophoresis was performed in a
VE-20 chamber (Helicon, Russia). Nonspecific esterases were fractionated in a $10 \%$ gel, whereas superoxide dismutases and malate dehydrogenases were fractionated in a $7.5 \%$ gel. Tris- $\mathrm{HCl}$ gel buffer was used for the electrophoresis (stacking gel buffer, $\mathrm{pH} 6.7$;

Table 1. Stenomphalia ravergiensis sampling sites

\begin{tabular}{c|l|l|l}
\hline No. & \multicolumn{1}{|c|}{ Site } & \multicolumn{1}{c|}{ Description } & \multicolumn{1}{c}{ Coordinates } \\
\hline 1 & Donets & $\begin{array}{l}\text { Floodplain of Severskii Donets River near the railway station. } \\
\text { Poplar thicket }\end{array}$ & $\begin{array}{l}50^{\circ} 35^{\prime} 52.13^{\prime \prime} \mathrm{N} \\
36^{\circ} 36^{\prime} 55.03^{\prime \prime} \mathrm{E}\end{array}$ \\
\hline 2 & Michurina & $\begin{array}{l}\text { Wasteland with ruderal vegetation } \\
\text { near Belenergomash production plant }\end{array}$ & $\begin{array}{l}50^{\circ} 37^{\prime} 0.71^{\prime \prime} \mathrm{N} \\
36^{\circ} 33^{\prime} 33.40^{\prime \prime} \mathrm{E}\end{array}$ \\
\hline 3 & Kalinina & $\begin{array}{l}\text { Territory of Belgorod Construction Material Combine (BCMC) } \\
\text { near a chalky slope }\end{array}$ & $\begin{array}{l}50^{\circ} 36^{\prime} 34.62^{\prime \prime} \mathrm{N} \\
36^{\circ} 36^{\prime} 33.79^{\prime \prime} \mathrm{E}\end{array}$ \\
\hline 4 & Botanicheskii sad & Maple thicket near the botanical garden of Belgorod State University & $\begin{array}{l}50^{\circ} 35^{\prime} 39.32^{\prime \prime} \mathrm{N} \\
36^{\circ} 33^{\prime} 17.07^{\prime \prime} \mathrm{E}\end{array}$ \\
\hline 5 & Vezelka & $\begin{array}{l}\text { Floodplain of Vezelka River near an area with private houses. } \\
\text { Moderately humid area with maple, poplar, and willow thicket }\end{array}$ & $\begin{array}{l}50^{\circ} 35^{\prime} 38.70^{\prime \prime} \mathrm{N} \\
36^{\circ} 33^{\prime} 52.36^{\prime \prime} \mathrm{E}\end{array}$ \\
\hline 6 & Kar'er & $\begin{array}{l}\text { An open area with steppe vegetation between an area } \\
\text { with private houses and a chalk pit }\end{array}$ & $\begin{array}{l}50^{\circ} 37^{\prime} 35.90^{\prime \prime} \mathrm{N} \\
36^{\circ} 31^{\prime} 1.33^{\prime \prime} \mathrm{E}\end{array}$ \\
\hline 7 & Vodstroi & $\begin{array}{l}\text { Shaded site in the floodplain of Vezelka River along railway tracks. } \\
\text { Maple thicket. Burdock, nettle, and bennet in the herb tier }\end{array}$ & $\begin{array}{l}50^{\circ} 35^{\prime} 25.00^{\prime \prime} \mathrm{N} \\
36^{\circ} 34^{\prime} 3.54^{\prime \prime} \mathrm{E}\end{array}$ \\
\hline
\end{tabular}




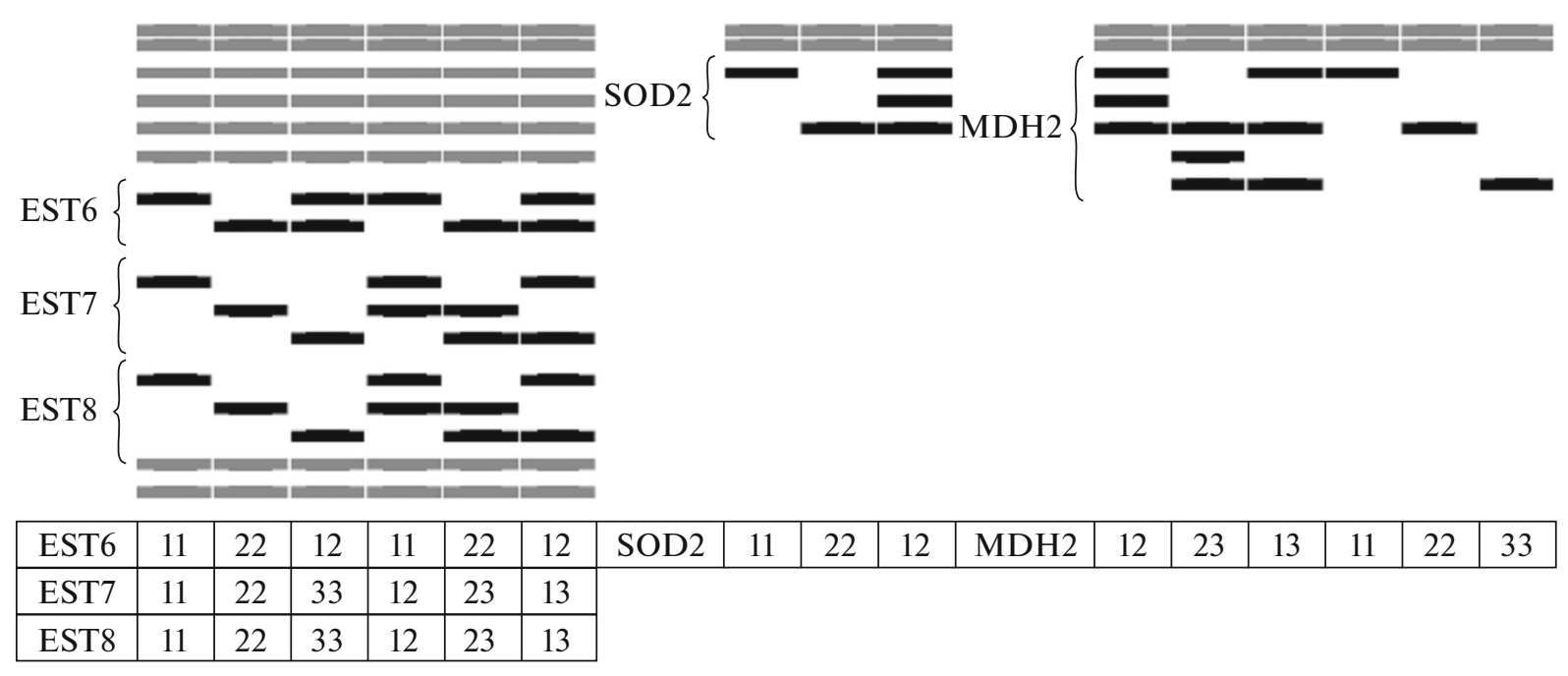

Fig. 2. Allozyme loci and the respective Stenomphalia ravergiensis genotypes (monomorphic loci are shown in gray).

resolving gel buffer, $\mathrm{pH}$ 8.9), and Tris-glycine buffer, $\mathrm{pH} 8.3$, was used as the running buffer. Gel block staining for nonspecific esterase detection was performed in a substrate mix of the following composition: Tris- $\mathrm{HCl}(\mathrm{pH} 7.4), \alpha$-naphthyl acetate, and Fast Red TR. The substrate mix for superoxide dismutase detection contained potassium phosphate buffer ( $\mathrm{pH}$ 7.8), NTS, and PMS, and that for malate dehydrogenase detection contained $0.1 \mathrm{M}$ Tris- $\mathrm{HCl}(\mathrm{pH} 8.4)$, sodium malate, NTS, PMS, and NAD.

Ten nonspecific esterase activity zones were detected in $S$. ravergiensis, and the three monomeric loci were EST6 (with two alleles), EST7, and EST8 (with three alleles each). We also identified one polymorphic locus of a dimeric superoxide dismutase SOD2 with two alleles and a dimeric malate dehydrogenase locus $M D H 2$ with three alleles (Fig. 2).

All loci mentioned above had a codominant type of inheritance. Locus boundaries were identified by comparing the empirical genotype distribution to the theoretically expected Hardy-Weinberg distribution; Pearson's $\chi^{2}$ test was used for the comparison (Table 2). GenALEx 6.501 software (Peakall and Smouse, 2012) was used for genetic data processing.

The samples used for the analysis of $S$. ravergiensis population viability were of a limited size and thus contained only a small part of the population allele pool. Therefore, we performed multilocus variability analysis. Multilocus combinations for each of the 175 individuals were calculated at the first step, and the total number of multilocus genotypes $\left(N_{\mathrm{MLG}}\right)$ and the number of unique multilocus genotypes $\left(N_{\mathrm{MLG}-1}\right)$, that is, combinations found in a single sample, were calculated at the following step. Multilocus genotype frequency distributions for each population were subsequently used to assess the potential genetic diversity expected upon an increase of sample size to infinity
(Nmax). The two nonparametric methods used for the analysis were the Chao1-bc (bias-corrected form for Chao1) (Chao, 2005) and the first-order jackknife method (Burnham and Overton, 1978). Multilocus genotype analysis was performed in the SPADE program (Chao and Shen, 2009).

Genetic distance calculation according to Nei and Li (1976), maximum-likelihood (ML) sample clustering, and bootstrap assessment (1000 iterations) were performed using TREECON software ver. 1.3b (Van de Peer and De Wachter, 1994).

\section{RESULTS AND DISCUSSION}

Allele frequencies and genetic variability parameters for the loci used are shown in Tables 3 and 4. The data obtained demonstrated a high level of allozyme locus variability in the groups studied. All the loci used were polymorphic $(P=100 \%)$ in two invasive groups (Botanicheskii sad and Vezelka) and in the population from the native range (Noratus).

Table 2. Comparison of genotype distribution to HardyWeinberg equilibrium in Stenomphalia ravergiensis colonies

\begin{tabular}{|c|c|c|c|c|c|c|c|c|}
\hline \multirow{2}{*}{ Locus } & \multicolumn{8}{|c|}{ Population } \\
\hline & 1 & 2 & 3 & 4 & 5 & 6 & 7 & 8 \\
\hline Est6 & Ns & Mono & Ns & Ns & Ns & Mono & Ns & $D^{*}$ \\
\hline Est 7 & Ns & Ns & $\mathrm{D}^{* * *}$ & $\mathrm{D}^{*}$ & $\mathrm{D}^{* *}$ & $\mathrm{D}^{* * *}$ & $\mathrm{D}^{*}$ & Ns \\
\hline Est8 & Mono & Mono & Mono & $\mathrm{D}^{* *}$ & Ns & Mono & Mono & Ns \\
\hline SOD2 & Ns & Mono & Ns & Ns & Ns & Mono & Ns & Ns \\
\hline$M D H 2$ & Ns & Ns & Ns & $\mathrm{D}^{*}$ & Ns & Ns & Ns & Ns \\
\hline
\end{tabular}

Mono-monomorphic locus; D-significant difference between the actual and theoretically predicted allele frequency distribution; $*_{-}-p<0.05 ; *^{*}-p<0.01 ; * * *-p<0.001$; ns_-no significant difference between the allele frequency distributions, $p>0.05$. 
Table 3. Allele frequencies for allozyme loci in Stenomphalia ravergiensis populations

\begin{tabular}{l|c|c|c|c|c|c|c|c|c}
\hline \multirow{2}{*}{ Locus } & \multirow{2}{*}{ Allele } & \multicolumn{7}{|c|}{ Populations } \\
\cline { 3 - 9 } & & 1 & 2 & 3 & 4 & 5 & 6 & 7 & 8 \\
\hline \multirow{2}{*}{ EST6 } & 1 & 0.100 & 0.000 & 0.080 & 0.480 & 0.360 & 0.000 & 0.420 & 0.309 \\
& 2 & 0.900 & 1.000 & 0.920 & 0.520 & 0.640 & 1.000 & 0.580 & 0.691 \\
\hline \multirow{3}{*}{ EST7 } & 1 & 0.260 & 0.000 & 0.560 & 0.360 & 0.340 & 0.560 & 0.440 & 0.022 \\
& 2 & 0.280 & 0.200 & 0.100 & 0.160 & 0.160 & 0.100 & 0.000 & 0.478 \\
& 3 & 0.460 & 0.800 & 0.340 & 0.480 & 0.500 & 0.340 & 0.560 & 0.500 \\
\hline \multirow{3}{*}{ EST8 } & 1 & 0.000 & 0.000 & 0.000 & 0.120 & 0.020 & 0.000 & 0.000 & 0.000 \\
& 2 & 0.000 & 0.000 & 0.000 & 0.140 & 0.060 & 0.000 & 0.000 & 0.184 \\
& 3 & 1.000 & 1.000 & 1.000 & 0.740 & 0.920 & 1.000 & 1.000 & 0.816 \\
\hline \multirow{2}{*}{ SOD2 } & 1 & 0.860 & 1.000 & 0.820 & 0.480 & 0.760 & 1.000 & 0.800 & 0.978 \\
& 2 & 0.140 & 0.000 & 0.180 & 0.520 & 0.240 & 0.000 & 0.200 & 0.022 \\
\hline \multirow{3}{*}{ MDH2 } & 1 & 0.540 & 0.640 & 0.500 & 0.580 & 0.740 & 0.620 & 0.440 & 0.360 \\
& 2 & 0.320 & 0.360 & 0.500 & 0.320 & 0.260 & 0.380 & 0.560 & 0.574 \\
& 3 & 0.140 & 0.000 & 0.000 & 0.100 & 0.000 & 0.000 & 0.000 & 0.066 \\
\hline
\end{tabular}

Moreover, the level of actual and expected heterozygosity in the majority of invasive colonies was comparable to that in the aboriginal population. The group that lived near the chalk pit (Kar'er) and the colony found on the side of the road leading to the chalk pit (Michurina site) formed an exception.

The values of the inbreeding coefficient were rather low in all groups investigated. The average actual heterozygosity for all loci studied in all samples showed significant similarity to the theoretically expected value (Kruskal-Wallis test: $p>0.05$ for all groups). An excess of heterozygotes was observed for some loci in individual colonies, but this excess proved statistically insignificant. For instance, a slight excess of individuals heterozygous for EST6, SOD2, and $M D H 2$ loci was identified in the Donets group, and a slight excess of heterozygotes for $E S T 7$ and $M D H 2$ loci was identified in the Michurina group, and therefore the inbreeding coefficient value for these groups was negative. All these data are indicative of high viability and genetic plasticity of $S$. ravergiensis, as expected for an invasive species (Barrett and Richardson, 1986).

The results of molecular analysis of variance (AMOVA) for the Belgorod colonies are shown in Table 5 (the Caucasian population was not included in this analysis). The data demonstrate the predomination of intrapopulational variance over interpopulational variance in the sample studied, this being indicative of weak genetic differentiation of the colonies analyzed (Wrait, 1940).

Importantly, genetic variability at the individual level predominated in the invasive groups. This was evident from the inbreeding coefficient for the individual relative to the large population (Fit $=0.216, p=$
0.001), being almost two times higher than the inbreeding coefficients for the individual relative to the subpopulation (Fis $=0.111, p=0.001$ ) and for the subpopulation relative to the large population ( $F s t=$ $0.117, p=0.001$ ).

Analysis of pairwise matrices for gene flow values $(\mathrm{Nm})$ and geographical distances between the snail groups investigated $(D g)$ did not reveal a significant correlation between these parameters, as is evident from a rather low value of the Mantel correlation coefficient $(0.375, p=0.084)$ (Mantel, 1967; José et al., 2013). Thus, the absence of a correlation between the spatial structure of the population and its genetic structure is likely (Fig. 3).

Deviation from the model of isolation by distance is observed in this case. The structure of expansion of the invasive species under investigation is more likely to correspond to the island model (Wrait, 1943), which implies migrant exchange between multiple daughter populations and a parent population (at an equal exchange rate).

As evident from Table 6, the genetic distance $(D)$ inferred from allozyme loci frequencies as described by Nei (1975) is rather low for the populations studied, and this corroborates poor genetic differentiation of the populations.

This assumption is partially confirmed by maximum-likelihood (ML) cluster analysis (Fig. 4). Samples from the Vezelka and Botanicheskii sad sites belong to the same cluster, and the Donets group is close to them. The Michurina and Kar'er groups belong to a separate cluster, and the Kalinina group is the closest to this cluster. 


\begin{tabular}{|c|c|c|c|c|c|c|c|c|c|c|c|c|c|c|c|c|c|c|c|c|c|c|c|}
\hline de & \multicolumn{6}{|c|}{$\begin{array}{l}\stackrel{1}{1} \\
+1 \\
\infty\end{array}$} & \multicolumn{6}{|c|}{$\begin{array}{l}\stackrel{d}{+} \\
+1 \\
+ \\
+\end{array}$} & \multicolumn{5}{|c|}{$\begin{array}{l}\stackrel{\Upsilon}{1} \\
+1 \\
\infty\end{array}$} & \multicolumn{6}{|c|}{ ¿ } \\
\hline 4 & $\begin{array}{c}\Xi \\
\vdots \\
\end{array}$ & $\begin{array}{l}\tilde{n} \\
\stackrel{n}{0}\end{array}$ & 1 & $\begin{array}{l}\hat{\sigma} \\
0 \\
0 \\
\dot{1}\end{array}$ & $\begin{array}{l}\text { సे } \\
\text { î }\end{array}$ & $\begin{array}{l}n \\
\hat{0} \\
0 \\
+1 \\
+1 \\
\hat{0} \\
0 \\
0 \\
1\end{array}$ & 1 & 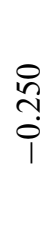 & 1 & 1 & \begin{tabular}{l}
$\stackrel{n}{n}$ \\
\multirow{2}{0}{} \\
$i$
\end{tabular} & $\begin{array}{l}\bar{\Xi} \\
\dot{0} \\
+1 \\
m \\
\tilde{N} \\
\tilde{0} \\
i\end{array}$ & $\begin{array}{l}0 \\
\infty \\
0 \\
0 \\
1\end{array}$ & $\begin{array}{l}\bar{r} \\
\ddot{n}\end{array}$ & $1 \stackrel{\bar{n}}{0}$ & $\begin{array}{l}\text { o } \\
0 \\
\dot{0}\end{array}$ & $\begin{array}{l}\stackrel{1}{0} \\
0 \\
+1 \\
0 \\
0 \\
0\end{array}$ & $\begin{array}{l}\infty \\
\tilde{o} \\
0 \\
0\end{array}$ & $\begin{array}{l}\underset{+}{\infty} \\
\text { ஸे } \\
0\end{array}$ & 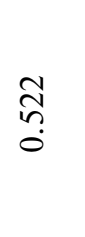 & 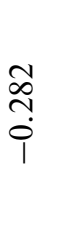 & $\begin{array}{l}\stackrel{+}{2} \\
\stackrel{0}{0}\end{array}$ & $\begin{array}{l}n \\
n \\
0 \\
+1 \\
0 \\
0 \\
0 \\
0 \\
0\end{array}$ \\
\hline \pm & $\stackrel{\infty}{\stackrel{\infty}{0}}$ & $\begin{array}{l}\text { Jै } \\
0 \\
0\end{array}$ & $\begin{array}{l}8 \\
8 \\
0\end{array}$ & $\underset{\Xi}{\mathbb{J}}$ & $\begin{array}{l}0 \\
\infty \\
? \\
0\end{array}$ & 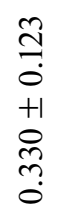 & 8 & $\begin{array}{l}\text { ते } \\
\text { ஸे }\end{array}$ & $\begin{array}{l}8 \\
8 \\
0\end{array}$ & $\begin{array}{l}8 \\
\vdots \\
0\end{array}$ & $\begin{array}{l}\overline{5} \\
\stackrel{0}{0}\end{array}$ & $\begin{array}{l}\infty \\
o \\
0 \\
0 \\
+1 \\
0 \\
0 \\
\stackrel{0}{0}\end{array}$ & 导 & $\begin{array}{l}\overrightarrow{0} \\
\ddot{n} \\
0\end{array}$ & 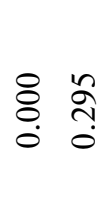 & $\begin{array}{l}8 \\
\text { ?ִ } \\
0\end{array}$ & $\begin{array}{l}n \\
\varrho \\
+1 \\
+1 \\
o n \\
0\end{array}$ & 高 & $\begin{array}{l}\underset{\nabla}{\sigma} \\
\stackrel{0}{0}\end{array}$ & $\stackrel{\infty}{\underset{\sigma}{0}}$ & $\stackrel{\text { वे }}{\circ}$ & $\begin{array}{l}\bar{n} \\
\tilde{n}\end{array}$ & $\begin{array}{l}2 \\
0 \\
0 \\
0 \\
+1 \\
0 \\
n \\
0\end{array}$ \\
\hline$\stackrel{8}{2}$ & 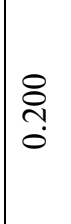 & 市 & $\begin{array}{l}8 \\
8 \\
0\end{array}$ & $\begin{array}{l}\stackrel{8}{\infty} \\
\text { ஸे } \\
0\end{array}$ & $\begin{array}{l}8 \\
\stackrel{8}{0}\end{array}$ & 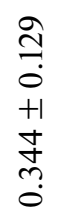 & $\begin{array}{l}0 \\
0 \\
0 \\
0\end{array}$ & o̊ & $\begin{array}{l}8 \\
8 \\
0 \\
0\end{array}$ & $\begin{array}{l}8 \\
8 \\
0 \\
0\end{array}$ & $\begin{array}{l}\text { ஜ̊ } \\
\stackrel{2}{0} \\
0\end{array}$ & 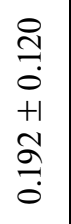 & $\begin{array}{l}0 \\
0 \\
0 \\
0\end{array}$ & 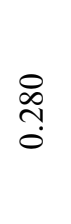 & 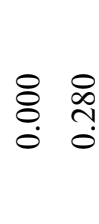 & $\begin{array}{l}\text { กิ } \\
\tilde{0}\end{array}$ & $\begin{array}{l}\mathscr{2} \\
\infty \\
0 \\
0 \\
+1 \\
+1 \\
\infty \\
\stackrel{+}{+} \\
0\end{array}$ & 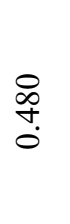 & $\stackrel{f}{+}$ & ્ֻત & $\begin{array}{l}\text { \&े } \\
\text { ب. } \\
0\end{array}$ & $\begin{array}{l}\stackrel{0}{0} \\
0 \\
0\end{array}$ & $\begin{array}{l}n \\
\infty \\
0 \\
0 \\
+1 \\
\infty \\
\infty \\
\infty \\
+ \\
0\end{array}$ \\
\hline- & $\begin{array}{l}n \\
\tilde{a} \\
0\end{array}$ & 吉 & $\begin{array}{l}8 \\
8 \\
0\end{array}$ & $\begin{array}{l}n \\
o \\
+ \\
0\end{array}$ & $\hat{a}$ & 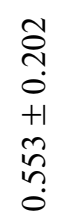 & $\begin{array}{l}8 \\
0 \\
0 \\
0\end{array}$ & $\begin{array}{l}8 \\
n \\
0\end{array}$ & $\begin{array}{l}8 \\
8 \\
0\end{array}$ & $\begin{array}{l}8 \\
8 \\
0 \\
0\end{array}$ & 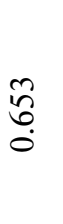 & $\begin{array}{l}\stackrel{m}{ \pm} \\
\vdots \\
+1 \\
\overrightarrow{0} \\
\tilde{0}\end{array}$ & 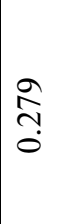 & $\begin{array}{l}\tilde{o} \\
\grave{o}\end{array}$ & 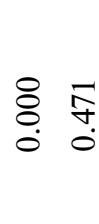 & 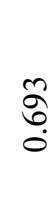 & $\begin{array}{l}8 \\
0 \\
0 \\
+1 \\
0 \\
\stackrel{0}{f} \\
0\end{array}$ & 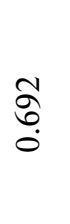 & $\stackrel{m}{a}$ & $\stackrel{n}{\stackrel{n}{0}}$ & 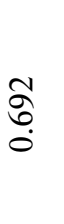 & $\bar{\sigma}$ & 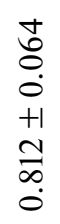 \\
\hline$\stackrel{*}{*}$ & 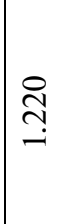 & $\begin{array}{l}\stackrel{2}{i} \\
\text { i }\end{array}$ & $\stackrel{8}{8}$ & $\stackrel{\bar{m}}{-}$ & $\underset{\substack{f \\
i}}{i}$ & $\begin{array}{l}\text { 令 } \\
\text { ? } \\
+1 \\
\stackrel{n}{n} \\
\stackrel{n}{=}\end{array}$ & 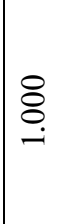 & $\stackrel{\text { 守 }}{-}$ & ه̊ & . & $\begin{array}{l}n \\
\infty \\
\infty \\
-\end{array}$ & 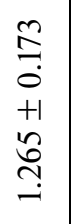 & $\stackrel{\Omega}{I}$ & 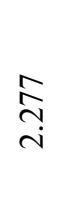 & $\stackrel{\circ}{\circ} \stackrel{\circ}{\nexists}$ & $\begin{array}{l}\stackrel{8}{0} \\
\text { i }\end{array}$ & 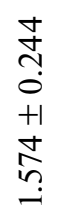 & $\hat{\alpha}$ & ڤ̊n & $\stackrel{2}{\stackrel{2}{ت}}$ & $\hat{\widehat{a}}$ & $\begin{array}{l}\stackrel{\infty}{\Xi} \\
\underset{\sim}{i}\end{array}$ & $\begin{array}{l}0 \\
\stackrel{1}{0} \\
0 \\
+1 \\
+1 \\
0 \\
\dot{0}\end{array}$ \\
\hline$\stackrel{8}{\pi}$ & $\begin{array}{l}8 \\
\vdots \\
i\end{array}$ & $\begin{array}{l}\stackrel{8}{8} \\
\dot{m}\end{array}$ & $\stackrel{8}{8}$ & $\begin{array}{l}\stackrel{8}{8} \\
i\end{array}$ & 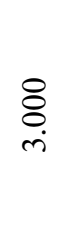 & 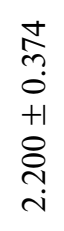 & 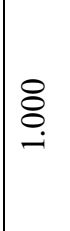 & $\underset{8}{\stackrel{0}{0}}$ & $\stackrel{8}{8}$ & ¿ & $\begin{array}{l}8 \\
8 \\
\text { ن }\end{array}$ & 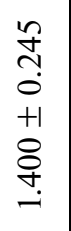 & $\begin{array}{l}8 \\
8 \\
i\end{array}$ & $\underset{\dot{\theta}}{\stackrel{8}{0}}$ & \begin{tabular}{ll}
$\&$ & $\&$ \\
\hdashline & $\vdots$ \\
\end{tabular} & $\begin{array}{l}\stackrel{8}{8} \\
i\end{array}$ & 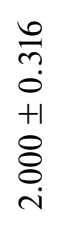 & $\begin{array}{l}8 \\
\vdots \\
i\end{array}$ & $\begin{array}{l}\stackrel{\Xi}{\dot{m}} \\
\dot{m}\end{array}$ & $\underset{\dot{\Xi}}{\stackrel{一}{ }}$ & $\begin{array}{l}8 \\
8 \\
i \\
\text { i }\end{array}$ & $\begin{array}{l}8 \\
\dot{8} \\
\dot{m}\end{array}$ & \begin{tabular}{l}
$n$ \\
\multirow{1}{+}{} \\
$\vdots$ \\
+1 \\
+1 \\
8 \\
0 \\
$i$ \\
$i$
\end{tabular} \\
\hline $\begin{array}{l}\mathscr{0} \\
0 \\
0 \\
0\end{array}$ & 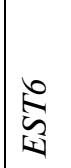 & $\hat{\hat{5}}$ & $\sum_{1}^{\infty}$ & 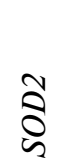 & 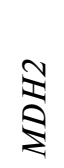 & 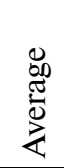 & $\begin{array}{l}6 \\
5 \\
5\end{array}$ & $\hat{E}$ & $\sum_{0}^{\infty}$ & 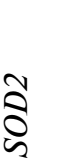 & $\underset{\mathbb{Z}}{\mathbb{Z}}$ & 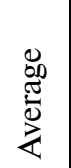 & 苟 & $\hat{5}$ & 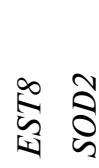 & $\underset{\Sigma}{\grave{Z}}$ & 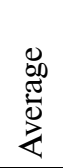 & है & $\hat{5}$ & $\sum_{i=1}^{\infty}$ & $\begin{array}{l}\tilde{\Xi} \\
\text { ธิ }\end{array}$ & $\underset{\Sigma}{\grave{Z}}$ & 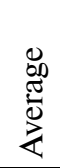 \\
\hline 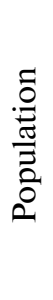 & & & - & & & & & & 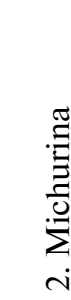 & & & & & & $\begin{array}{l}\stackrel{\widetilde{\Xi}}{\Xi} \\
: \widetilde{\Xi} \\
\tilde{\pi} \\
\dot{n}\end{array}$ & & & & & 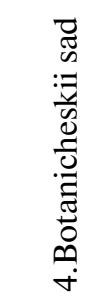 & & & \\
\hline
\end{tabular}


GENETIC STRUCTURE ANALYSIS OF THE ALIEN MOLLUSK

\begin{tabular}{|c|c|c|c|c|c|c|c|c|c|c|c|c|c|c|c|c|c|c|c|c|c|c|c|c|c|c|}
\hline $\begin{array}{l}\text { de } \\
\text { R }\end{array}$ & & & 8 & & & & & & \begin{tabular}{l}
\multirow{J}{*}{} \\
+1 \\
$\stackrel{+}{+}$
\end{tabular} & & & & & & $\begin{array}{l}\stackrel{\gamma}{2} \\
+1 \\
\infty\end{array}$ & & & & & & 으 & & & & $\begin{array}{l}\infty \\
\infty \\
\infty \\
+1 \\
0 \\
n\end{array}$ & 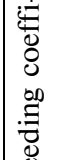 \\
\hline 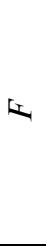 & $\stackrel{2}{2}$ & $\begin{array}{l}\stackrel{+}{\sigma} \\
\dot{0}\end{array}$ & $\begin{array}{l}\stackrel{0}{0} \\
\dot{0} \\
1\end{array}$ & $\stackrel{\dddot{I}}{\ddot{0}}$ & $\begin{array}{l}\text { ठे } \\
0 \\
0\end{array}$ & $\begin{array}{l}0 \\
\circ \\
0 \\
0 \\
+1 \\
+1 \\
\vdots \\
0 \\
0\end{array}$ & 1 & $\begin{array}{l}\text { D̊n } \\
\vdots \\
0\end{array}$ & 1 & 1 & $\begin{array}{l}0 \\
0 \\
0 \\
0\end{array}$ & 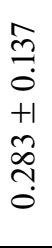 & $\begin{array}{l}\hat{\sigma} \\
0 \\
\dot{0}\end{array}$ & $\frac{m}{n}$ & 1 & $\begin{array}{l}8 \\
\stackrel{8}{8} \\
0\end{array}$ & 吕 & 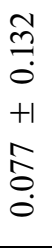 & $\underset{\stackrel{\sim}{+}}{\stackrel{\sim}{0}}$ & $\begin{array}{l}0 \\
0 \\
0 \\
0\end{array}$ & 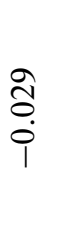 & 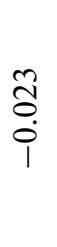 & $\frac{\overparen{\Xi}}{\stackrel{0}{i}}$ & $\begin{array}{l}\overrightarrow{0} \\
0 \\
0 \\
+1 \\
0 \\
0 \\
0 \\
0\end{array}$ & 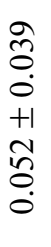 & 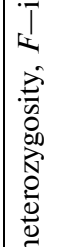 \\
\hline$\stackrel{\Sigma}{*}$ & $\begin{array}{l}\overrightarrow{0} \\
+ \\
0\end{array}$ & $\begin{array}{l}\hat{O} \\
\dot{0} \\
\dot{0}\end{array}$ & $\frac{\stackrel{P}{6}}{\dot{0}}$ & $\begin{array}{l}n \\
\tilde{0} \\
\tilde{n} \\
0\end{array}$ & $\begin{array}{l}n \\
\infty \\
n \\
0\end{array}$ & 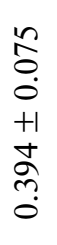 & $\begin{array}{l}8 \\
8 \\
0 \\
0\end{array}$ & $\begin{array}{l}\overline{0} \\
n \\
0\end{array}$ & $\begin{array}{l}8 \\
8 \\
0 \\
0\end{array}$ & $\begin{array}{l}8 \\
8 \\
0 \\
0\end{array}$ & $\underset{\nabla}{\nabla}$ & 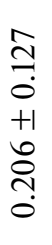 & $\begin{array}{l}\hat{\infty} \\
\dot{0}\end{array}$ & $\begin{array}{r}\stackrel{m}{\sigma} \\
\dot{0}\end{array}$ & $\begin{array}{l}8 \\
8 \\
8 \\
0\end{array}$ & ก্ণ & 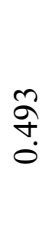 & 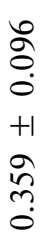 & 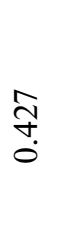 & $\stackrel{\vec{N}}{n}$ & $\begin{array}{l}\text { 尺) } \\
\text { గొ } \\
0\end{array}$ & $\underset{0}{\stackrel{m}{0}}$ & $\hat{n}$ & $\begin{array}{l}\sigma \\
0 \\
0 \\
+1 \\
0 \\
0 \\
0 \\
0\end{array}$ & 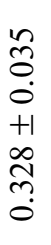 & 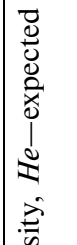 \\
\hline$\stackrel{8}{*}$ & $\begin{array}{l}8 \\
\stackrel{8}{+} \\
\stackrel{0}{0}\end{array}$ & ণ্ণি & $\frac{8}{0}$ & 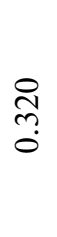 & $\begin{array}{l}0 \\
\text { గి } \\
0\end{array}$ & 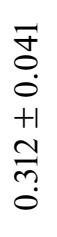 & $\begin{array}{l}8 \\
8 \\
0 \\
0\end{array}$ & $\begin{array}{l}\stackrel{\infty}{\infty} \\
\stackrel{0}{0}\end{array}$ & $\begin{array}{l}8 \\
8 \\
0 \\
0\end{array}$ & $\begin{array}{l}8 \\
8 \\
0 \\
0\end{array}$ & 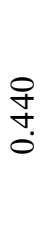 & 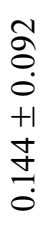 & $\begin{array}{l}\text { กิ } \\
\text { ก }\end{array}$ & $\underset{\substack{+0}}{\stackrel{+}{0}}$ & $\begin{array}{l}8 \\
8 \\
0 \\
0\end{array}$ & ণి & $\begin{array}{l}8 \\
\stackrel{0}{0} \\
? \\
0\end{array}$ & 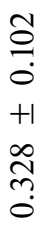 & 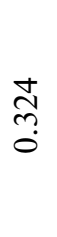 & ڤิ & 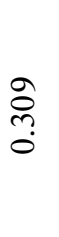 & $\begin{array}{l}\stackrel{+}{+} \\
0 \\
0\end{array}$ & $\begin{array}{l}\text { O̊̊ } \\
\stackrel{0}{0} \\
0\end{array}$ & 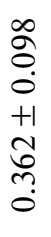 & 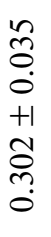 & 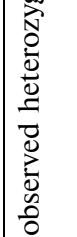 \\
\hline- & $\begin{array}{l}n \\
n \\
0 \\
0\end{array}$ & $\hat{8}$ & $\underset{\sim}{\stackrel{ \pm}{0}}$ & $\begin{array}{l}\tilde{n} \\
\tilde{n}\end{array}$ & ñ & 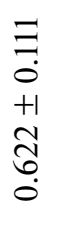 & $\begin{array}{l}8 \\
8 \\
0 \\
0\end{array}$ & 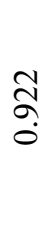 & $\begin{array}{l}8 \\
8 \\
0 \\
0\end{array}$ & $\begin{array}{l}8 \\
8 \\
0 \\
0\end{array}$ & $\begin{array}{l}\text { Jర } \\
\stackrel{0}{0} \\
0\end{array}$ & $\begin{array}{l}\infty \\
\stackrel{0}{0} \\
+1 \\
+1 \\
\stackrel{0}{0}\end{array}$ & $\begin{array}{l}\stackrel{0}{\infty} \\
0 \\
0 \\
0\end{array}$ & $\begin{array}{l}0 \\
\infty \\
0 \\
0\end{array}$ & $\begin{array}{l}8 \\
8 \\
0 \\
0\end{array}$ & \begin{tabular}{l}
$\stackrel{8}{8}$ \\
\hdashline \\
\hdashline
\end{tabular} & $\begin{array}{l}\stackrel{0}{0} \\
\& \\
0 \\
0\end{array}$ & $\begin{array}{l}\tilde{n} \\
? \\
+1 \\
= \\
\tilde{n}\end{array}$ & $\begin{array}{l}\infty \\
0 \\
0\end{array}$ & $\frac{\vec{\infty}}{\stackrel{\infty}{0}}$ & 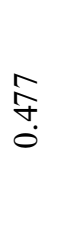 & $\begin{array}{l}0 \\
0 \\
0 \\
0\end{array}$ & $\begin{array}{l}0 \\
\infty \\
\infty \\
0\end{array}$ & \begin{tabular}{l}
$\stackrel{0}{0}$ \\
\hdashline \\
0 \\
+1 \\
0 \\
$n$ \\
0
\end{tabular} & $\begin{array}{l}n \\
2 \\
0 \\
0 \\
+1 \\
\bar{n} \\
0\end{array}$ & 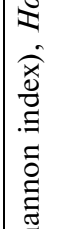 \\
\hline$\stackrel{0}{*}$ & $\begin{array}{l}n \\
n \\
\infty \\
-1\end{array}$ & $\begin{array}{l}\text { ڤn } \\
\stackrel{n}{n} \\
\text { in }\end{array}$ & 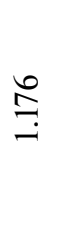 & 志 & ڤֶ) & 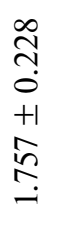 & $\underset{8}{8}$ & $\stackrel{\hat{N}}{\stackrel{N}{i}}$ & ஓ̊ & ஓ & ळ人 & 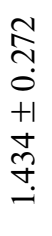 & $\stackrel{\circ}{2}$ & 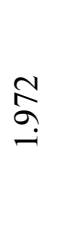 & ஓ & 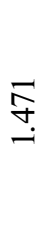 & $\stackrel{N}{\widehat{\alpha}}$ & 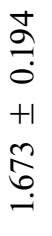 & $\stackrel{n}{\stackrel{2}{+}}$ & $\begin{array}{l}\infty \\
\infty \\
\infty \\
ن\end{array}$ & $\stackrel{\text { ঙे }}{\stackrel{\sim}{-}}$ & $\stackrel{n}{\text { fn }}$ & $\frac{\stackrel{a}{n}}{i}$ & $\begin{array}{l}\infty \\
\stackrel{0}{0} \\
0 \\
+1 \\
0 \\
\hat{\sigma} \\
\stackrel{-}{-}\end{array}$ & 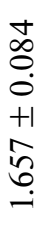 & 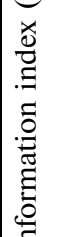 \\
\hline$\stackrel{\nabla}{\nabla}$ & $\begin{array}{l}8 \\
8 \\
\text { i }\end{array}$ & 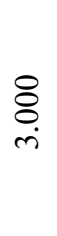 & 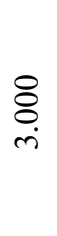 & $\begin{array}{l}\stackrel{8}{8} \\
\text { i }\end{array}$ & $\begin{array}{l}8 \\
8 \\
0 \\
\text { ¿i }\end{array}$ & 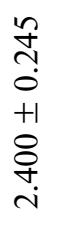 & $\underset{8}{8}$ & $\begin{array}{l}8 \\
\dot{8} \\
\dot{m}\end{array}$ & 8̊ & ஓ̊ & $\begin{array}{l}8 \\
8 \\
\text { ㄱ. }\end{array}$ & $\begin{array}{l}8 \\
8 \\
+ \\
0 \\
+1 \\
8 \\
8 \\
0 \\
\end{array}$ & $\begin{array}{l}8 \\
8 \\
\stackrel{8}{ }\end{array}$ & $\begin{array}{l}8 \\
8 \\
0 \\
\text { i }\end{array}$ & $\underset{8}{8}$ & $\begin{array}{l}\stackrel{8}{8} \\
\text { i }\end{array}$ & $\begin{array}{l}8 \\
8 \\
\text { ¿ }\end{array}$ & 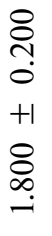 & $\begin{array}{l}8 \\
8 \\
\text { ¿ } \\
\text { i }\end{array}$ & $\begin{array}{l}8 \\
\varnothing \\
\dot{\delta}\end{array}$ & $\begin{array}{l}8 \\
8 \\
\text { ¿ }\end{array}$ & $\begin{array}{l}8 \\
8 \\
\text { ¿ }\end{array}$ & $\begin{array}{l}8 \\
\varnothing \\
\dot{\delta}\end{array}$ & 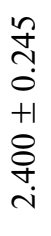 & $\begin{array}{l}9 \\
= \\
0 \\
+1 \\
0 \\
0 \\
\dot{n} \\
ن\end{array}$ & 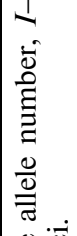 \\
\hline 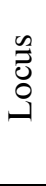 & $\frac{6}{5}$ & $\hat{5}$ & 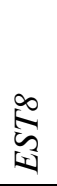 & $\stackrel{\overbrace{}}{\widehat{\sigma}}$ & $\underset{\mathbb{Z}}{\mathbb{Z}}$ & 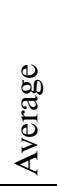 & $\underbrace{0}_{0}$ & $\hat{5}$ & $\sum_{5}^{\infty}$ & ิิ & 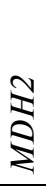 & 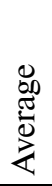 & $\underbrace{6}_{5}$ & $\hat{5}$ & $\sum_{-1}^{\infty}$ & ڤิ & $\underset{Z}{\overparen{Z}}$ & 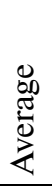 & $\frac{6}{5}$ & $\hat{5}$ & $\sum_{5}^{\infty}$ & 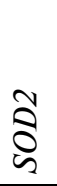 & $\underset{\precsim}{\grave{Z}}$ & $\begin{array}{l}0 \\
0 \\
\frac{0}{0} \\
0 \\
⿱ 亠 乂\end{array}$ & & 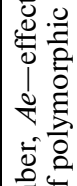 \\
\hline 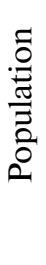 & & & $\begin{array}{l}\frac{\pi}{\tilde{J}} \\
\mathbb{N} \\
\stackrel{D}{D} \\
\text { in }\end{array}$ & & & & & & $\begin{array}{l}\dot{0} \\
\dot{\Xi} \\
\dot{U} \\
\dot{0}\end{array}$ & & & & & & $\begin{array}{l}\overline{0} \\
\bar{n} \\
\bar{n} \\
0 \\
> \\
N\end{array}$ & & & & & & 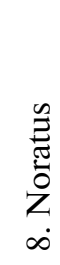 & & & & 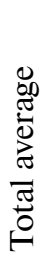 & 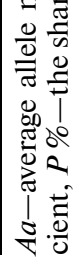 \\
\hline
\end{tabular}


Table 5. Results of molecular variance analysis for invasive Stenomphalia ravergiensis populations

\begin{tabular}{l|c|c|c|c|c|c|c|c}
\hline Variability source & $d f$ & $S S$ & $M S$ & Est. Var. & $\%$ & Fst & Fis & Fit \\
\hline Intrapopulational & 6 & 38.506 & 6.418 & 0.110 & 12 & & & \\
Interpopulational & 168 & 154.240 & 0.918 & 0.092 & 10 & & 0.117 & 0.111 \\
Interindividual & 175 & 128.500 & 0.734 & 0.734 & 78 & & 0.216 \\
Total & 349 & 321.246 & & 0.936 & 100 & & & \\
\hline
\end{tabular}

Fit-inbreeding coefficient for the individual relative to the large population, Fis-inbreeding coefficient for the individual relative to the subpopulation, $F s t$-inbreeding coefficient for the subpopulation relative to the large population; $p=0.001$.

Table 6. Pairwise genetic distance $(D)$ estimates for Stenomphalia ravergiensis populations

\begin{tabular}{c|c|c|c|c|c|c|c|c}
\hline Populations & 1 & 2 & 3 & 4 & 5 & 6 & 7 & 8 \\
\hline 1 & 0.000 & & & & & & & \\
2 & 0.031 & 0.000 & & & & & & \\
3 & 0.029 & 0.084 & 0.000 & & & & & \\
4 & 0.118 & 0.190 & 0.122 & 0.000 & & & & \\
5 & 0.039 & 0.075 & 0.055 & 0.045 & 0.000 & & & \\
6 & 0.028 & 0.067 & 0.012 & 0.174 & 0.064 & 0.000 & & \\
7 & 0.066 & 0.105 & 0.047 & 0.071 & 0.039 & 0.077 & 0.000 & \\
8 & 0.060 & 0.068 & 0.111 & 0.168 & 0.097 & 0.117 & 0.094 & 0.000 \\
\hline
\end{tabular}

The first $S$. ravergiensis individuals found in the town of Belgorod were collected from the Vodstroi site (Snegin and Prisnyi, 2008), and therefore one may assume that further expansion of the species started at this site and proceeded along the Vezelka River and onto the botanical garden territory. Small privately owned houses and vegetable patches occupy a major part of this area, and this may underlie the expansion of mollusks together with plant debris. Genetic remoteness of the other more monomorphic groups from a different cluster can be a consequence of gene drift evoked by the transport of a small number of $S$. ravergiensis individuals into the industrial area from the primary introduction site. All sites mentioned above are located in the industrial area and connected by roads. However, a second independent introduction of $S$. ravergiensis into Belgorod cannot be ruled out.

The results of multilocus genotype analysis are shown in Table 7. Data of multilocus variability analysis are in agreement with the results presented above. Multilocus variability was the lowest in the Kar'er and Michurina groups, this being due to the high share of monomorphic loci. The number of multilocus genotypes was the largest in colonies from the Botanicheskii sad and Vezelka sites. The nonparametric Chao1-bc method showed that the potential genetic diversity was higher in these groups than in the aboriginal population from Armenia.

Notably, the data obtained by us are somewhat in agreement with the regularities identified for other invasive mollusk species. For instance, genetic differentiation between invasive populations of such species often turns out to be insignificant. This phenomenon was thoroughly characterized in aquatic mollusks. For instance, high genetic similarity between the colonies was observed in the studies of Dreissenidae invasions (Marsden and Spidle, 1996; Voroshilova, 2015). Studies of Eurasian and North American populations of two species of the genus Dreissena revealed high genetic diversity and the absence of a "founder effect" in the invasive populations of both species. However, the genetic structure of invasive populations changed with time, with dissimilar changes observed in different species studied (Brown and Stepien, 2010). Studies of the populations of another invasive mollusk, Perna viridis, did not reveal any differences between the invasive populations, but all invasive groups differed from the native ones. Moreover, the genetic diversity of invasive groups was lower, in contrast to the observa-

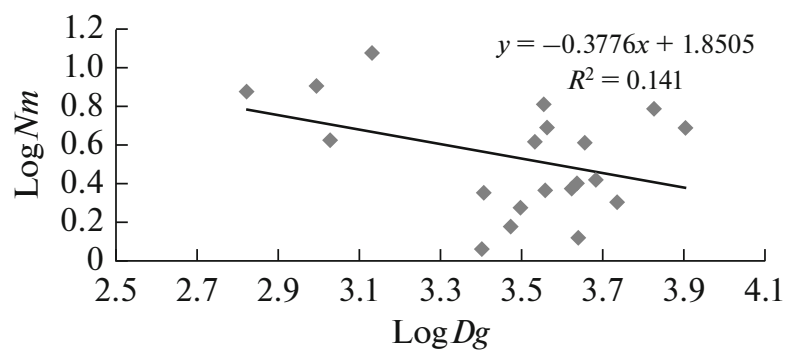

Fig. 3. Linear regression of gene flow logarithm $\mathrm{Nm}$ between pairs of Stenomphalia ravergiensis populations with regard to the logarithm $D g$ of the geographic distance between the populations. 


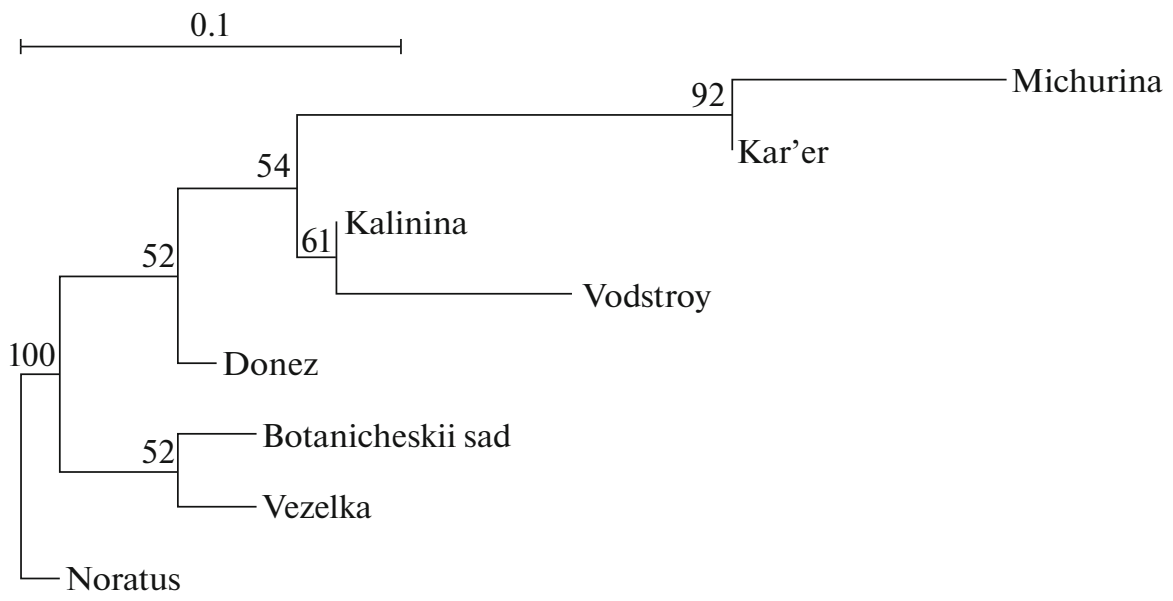

Fig. 4. Maximum-likelihood (ML) dendrogram of genetic distances between Stenomphalia ravergiensis groups. The share of populations grouped after the bootstrap test is shown at the nodes.

tions made in most native populations (Gilg et al., 2012). Slight differences between the inbreeding coefficients of invasive and native populations were reported for the freshwater mollusk Physa acuta (Bousset et al., 2004). A study of the population of the bivalve mollusk Potamocorbula amurensi revealed high genetic diversity in the subpopulations, whereas the population as a whole was genetically uniform (Duda, 1994). Allozyme analysis of invasive populations of the terrestrial snail Theba pisana in Australia revealed considerable reduction of genetic diversity (Johnson, 1988).

Allozyme marker-based studies of continuous and ephemeral populations of the invasive species Brephulopsis cylindrica in the town of Nikolaev (Ukraine) also showed that small isolated populations of this terrestrial mollusk (including urbanized populations) tended toward a decrease in genetic diversity related to the occurrence of stochastic genetic processes (gene drift or founder effect) (Kramarenko and Snegin, 2015). However, the intragroup variability decrease was accompanied by interpopulation distance increase in this species $(F \mathrm{st}=0.281)$, this being in agreement with the shifting balance theory of evolution (Wright, 1970). This theory implies an increase in subdivided metapopulation variability concomitantly to a decrease in subpopulation heterogeneity.

The concluding stage of our study involved effective size calculation for the colonies investigated. The formula used took the inbreeding level in the population into account $(\mathrm{Li}, 1976)$ :

$$
N e=\frac{N}{1+F}
$$

where $N e$ is the effective population size, $N$ is the number of individuals in the sample, and $F$ is the inbreeding coefficient.

Table 7. The number of multilocus genotypes identified and estimates of potential genetic diversity for Stenomphalia ravergiensis population studied

\begin{tabular}{|c|c|c|c|c|c|c|}
\hline \multirow{3}{*}{ Population } & \multirow{3}{*}{$N_{\mathrm{MLG}}$} & \multirow{3}{*}{$N_{\mathrm{MLG}-1}$} & \multicolumn{4}{|c|}{ Method } \\
\hline & & & \multicolumn{2}{|c|}{ Chao1-bc } & \multicolumn{2}{|c|}{ first-order jackknife } \\
\hline & & & $N \max \pm S E$ & $95 \% \mathrm{CI}$ & $N \max \pm S E$ & $95 \% \mathrm{CI}$ \\
\hline Botanicheskii sad & 23 & 19 & $90.2 \pm 44.4$ & $43.6-241.8$ & $43.2 \pm 6.3$ & $34.1-59.6$ \\
\hline Vezelka & 22 & 13 & $113.2 \pm 62.6$ & $49.0-330.4$ & $41.2 \pm 6.1$ & $32.4-57.4$ \\
\hline Vodstroi & 20 & 7 & $48.8 \pm 20.1$ & $28.4-119.2$ & $27.8 \pm 50.3$ & $34.4-72.3$ \\
\hline Donets & 20 & 11 & $36.8 \pm 11.7$ & $24.9-77.6$ & $34.4 \pm 5.3$ & $21.1-49.0$ \\
\hline Kalinina & 16 & 5 & $33.6 \pm 14.5$ & $20.3-88.2$ & $26.6 \pm 4.5$ & $20.7-39.7$ \\
\hline Kar'er & 10 & 0 & $12.9 \pm 4.0$ & $10.4-32.2$ & $13.8 \pm 2.7$ & $11.1-23.5$ \\
\hline Michurina & 5 & 0 & $5.0 \pm 0.0$ & 5.0 & $5.0 \pm 0.0$ & 5.0 \\
\hline Noratus & 34 & 21 & $57.4 \pm 14.1$ & $41.9-103.6$ & $53.7 \pm 6.3$ & $44.7-70.2$ \\
\hline
\end{tabular}

$N_{\mathrm{MLG}}$-total number of multilocus genotype variants identified; $N_{\mathrm{MLG}-1}$-number of unique variants of multilocus genotypes; $N m a x-$ potential genetic variability expected upon an increase in sample size to infinity. 
Table 8. Effective size of Stenomphalia ravergiensis colonies

\begin{tabular}{l|r|c|c|c|c|c|c|c}
\hline \multirow{2}{*}{ Parameter } & \multicolumn{9}{c}{ Colonies } \\
\cline { 2 - 9 } & 1 & 2 & 3 & 4 & 5 & 6 & 7 & 8 \\
\hline $\mathrm{Ne}$ & 25 & 25 & 22.6 & 23.4 & 21.8 & 19.5 & 23.2 & 24.7 \\
$\mathrm{Ne} / \mathrm{N}$ & 1 & 1 & 0.904 & 0.936 & 0.872 & 0.780 & 0.928 & 0.988 \\
\hline
\end{tabular}

Importantly, this formula implies that inbreeding coefficient values range from 0 to 1 . Therefore, we assigned a zero value of the inbreeding coefficient $F$ to some populations which were shown to have negative values of this coefficient.

We calculated the ratio between the effective sample size and total sample size in order to obtain comparable data (Table 8). The average $\mathrm{Ne} / \mathrm{N}$ value derived from the data obtained was $0.917 \pm 0.029$, this being within the overall range of relative $\mathrm{Ne}$ suggested by Crow, Morton, and Kimura (Crow and Morton, 1955; Crow and Kimura, 1970). These researchers showed that the average value of relative $\mathrm{Ne}$ was 0.75 for most organisms. Moreover, the average relative $\mathrm{Ne}$ calculated for Caucasian snail was significantly higher (at $p=0.05$ ) than the analogous values for the aboriginal reference species of terrestrial mollusks from the study site (the value for Bradybaena fruticum was $0.800 \pm$ 0.021 (data for 40 populations) and that for Chondrula tridens was $0.661 \pm 0.013$ (data for 25 populations)) (Snegin, 2012a, 2012b) and similar to the value for the thriving invasive species Helix pomatia $(0.870 \pm 0.043$; data for 12 populations) (Snegin and Artemchuk, 2016), this being an indirect indication of a high viability level of $S$. ravergiensis populations studied.

\section{CONCLUSIONS}

Thus, our results point at the high viability of invasive $S$. ravergiensis colonies manifested as gene pool richness and high values of effective population size. A high share of individual variability provides for genetic plasticity, which is known (Lee, 2002) to promote further development of the invasion process.

\section{ACKNOWLEDGMENTS}

We are grateful to M.V. Arzumanyan, who kindly provided material for analysis.

\section{REFERENCES}

Akramovskii, N.N., Fauna Armyanskoi SSR. Mollyuski (Mollusca) (The Fauna of the Armenian SSR. Mollusca), Yerevan: Akad. Nauk ArmSSR, 1976.

Alimov, A.F. and Bogutskaya, N.G., Biologicheskie invazii v vodnykh $i$ nazemnykh ekosistemakh (Biological Invasions in Aquatic and Terrestrial Ecosystems), Moscow: KMK, 2004.

Balashov, I.A., Kramarenko, S.S., Zhukov, A.V., Shklyaruk, A.N., Baidashnikov, A.A., and Vasyliuk, A.V.,
Contribution to the knowledge of terrestrial mollusks in southeastern Ukraine, Malacol. Bohemoslov., 2013, vol. 12 , pp. 62-69.

Barrett, S.C.H. and Richardson, B.J., Genetic attributes of invading species, in Ecology of Biological Invasions: An Australian Perspective, Groves, R. and Burdon, J.J., Eds., Canberra: Aust. Acad. Sci., 1986, pp. 21-33.

Bousset, L., Henry, P.-Y., Sourroulle, P., and Jarne, P., Population biology of the invasive freshwater snail Physa acuta approached through genetic markers, ecological characterization and demography, Mol. Ecol., 2004, vol. 13, pp. 2023-2036.

Brown, J.E. and Stepien, C.A., Population genetic history of the dreissenid mussel invasions: expansion patterns across North America, Biol. Invasions, 2010, vol. 12, no. 11 , pp. $3687-3710$.

Burnham, K.P. and Overton, W.S., Estimation of the size of a closed population when capture probabilities vary among animals, Biometrika, 1978, vol. 65, pp. 625-633.

Chao, A., Species richness estimation, in Encyclopedia of Statistical Science, Balakrishnan, N., Read, C.B., and Vidakovic, B., Eds., New York: Wiley, 2005, pp. 79077916.

Chao, A. and Shen, T.J., SPADE, Anne Chao's Website, 2009 (Electronic Document). http://chao.stat.nthu.edu.tw. Assessed November 16, 2015.

Clarke, B., Murray, J., and Johnson, M.S., The extinction of endemic species by a program of biological control, Pacific Sci., 1984, vol. 38, pp. 97-104.

Coote, T. and Loeve, E., From 61 species to five: endemic tree snails of the Society Islands fall prey to an illjudged biological control programme, Oryx, 2003, vol. 37, pp. 91-96.

Crow, J.F. and Kimura, M., An Introduction to Population Genetics Theory, New York: Harpers and Row, 1970.

Crow, J.F. and Morton, N.E., Measurement of gene frequency drift in small population, Evolution, 1955, vol. 9, pp. 202-214.

Diniz-Filho, J.A., Soares, T.N., Lima, J.S., Dobrovolski, R., Landeiro, V.L., de Campos Telles, M.P., Rangel, T.F., and Bini, L.M., Mantel test in population genetics, Genet. Mol. Biol., 2013, vol. 36, no. 4, pp. 475-485.

Duda, T.F., Jr., Genetic population structure of the recently introduced Asian clam, Potamocorbula amurensis, in San Francisco Bay, Mar. Biol., 1994, vol. 119, pp. 235-241.

Ghalambor, C.K., McKay, J.K., Carrol, S.P., and Reznick, D.N., Adaptive versus non-adaptive phenotypic plasticity and the potential for contemporary adaptation in new environments, Funct. Ecol., 2007, vol. 21, pp. 394-407. 
Gilg, M.R., Johnson, E.G., Gobin, J., Bright, B.M., and Ortolaza, A.I., Population genetics of introduced and native populations of the green mussel, Perna viridis: determining patterns of introduction, Biol. Invasions, 2012 (Electronic Document). Assessed August 2, 2012. doi 10.1007/s10530-012-0301-2

Johnson, M.S., Founder effects and geographic variation in the land snail Theba pisana, Heredity, 1988, vol. 61, pp. 133-142.

Kiyashko, P.V., Features of the highland malacofauna of the Western Caucasus, Mater. VII soveshchaniya po izucheniyu mollyuskov "Mollyuski: Morfologiya, taksonomiya, biogeografiya i ekologiya" (Proc. VII Meet. on the Study of Mollusks "Mollusks: Morphology, Taxonomy, Biogeography, and Ecology"), Egorov, E.N., Kiyashko, P.V., and Sirenko, B.I., Eds., St. Petersburg: Zool. Inst., Ross. Akad. Nauk, 2007, pp. 124-127.

Kramarenko, S.S. and Snegin, E.A., Genetic structure of the continuous and ephemeral populations of the land snail Brephulopsis cylindrica (Gastropoda, Pulmonata, Enidae), Russ. J. Genet.: Appl. Res., 2015, vol. 5, no. 5, pp. 469-478.

Lee, C.E., Evolutionary genetics of invasive species, TRENDS Ecol. Evol., 2002, vol. 17, no. 8, pp. 386-391.

Lee, C.E. and Gelembiuk, G.W., Evolutionary origins of invasive populations, Evol. Appl., 2008, vol. 1, no. 3, pp. 427-448.

Li, Ch.Ch., First Course in Population Genetics, Pacific Grove: Boxwood, 1976.

Mantel, N., The detection of disease clustering and a generalized regression approach, Cancer Res., 1967, vol. 27, pp. 209-220.

Marsden, J.E., Spidle, A.P., and May, B., Review of genetic studies of Dreissena spp., Am. Zool., 1996, vol. 36, no. 3, pp. 259-270.

Mayr, E., Change of genetic environment and evolution, in Evolution as a Process, Huxley, J., Hardy, A.C., and Ford, E.B., Eds., London: Allen \& Unwin, 1954, pp. $157-180$.

Murray, J., Murray, E., Johnson, M.S., and Clarke, B., The extinction of Partula on Moorea, Pacific Sci., 1988, vol. 42, pp. 150-153.

Nei, M., Molecular population genetics and evolution, Front. Biol., 1975, vol. 40.

Nei, M. and Li, W.H., Mathematical model for studying genetic variation in terms restriction endonucleases, Proc. Natl. Acad. Sci. USA, 1979, vol. 76, pp. 52695273.

Parker, I.M., Simberloff, D., Lonsdale, W.M., Goodell, K., Wonham, M., Kareiva, P.M., Williamson, M.H., von Holle, B., Moyle, P.B., Byers, J.E., and Goldwasser, L., Impact: toward a framework for understanding the ecological effects of invaders, Biol. Invasions, 1999, vol. 1, no. 1, pp. 3-19.
Peakall, R. and Smouse, P.E., GenALEx 6.5: genetic analysis in Excel. Population genetic software for teaching and research-an update, Bioinformatics, 2012, vol. 28, pp. 2537-2539.

Shileiko, A.A., Terrestrial mollusks of the superfamily Helicoidea, in Fauna SSSR: Mollyuski (Fauna of the Soviet Union: Mollusks), Leningrad: Nauka, 1978, vol. 3, no. 6.

Snegin, E.A., The genetic structure of model species populations of terrestrial mollusks in conditions of urbanized landscape using the example of Chondrula tridens Müll (Gastropoda, Pulmonata), Russ. J. Genet.: Appl. Res., 2012, vol. 2, no. 2, pp. 160-170.

Snegin, E.A., Spatial and temporal aspects of the ecological and genetic structure of invertebrate animal populations (on example of terrestrial mollusks and insects of the south of the Central Russian Upland), Extended Abstract of Doctoral (Biol.) Dissertation, Belgorod, 2012.

Snegin, E.A. and Adamova, V.V., Analysis of demographic and morphological parameters of populations of adventitious mollusks Stenomphalia ravergiensis (Mollusca, Gastropoda, Pulmonata) in the conditions of the south of the Central Russian Upland, Vestn. Tomsk. Gos. Univ., Biol., 2016, no. 3 (35), pp. 149-160.

Snegin, E.A. and Artemchuk, O.Yu., Morphogenetic analysis of Helix pomatia L. (Pulmonata, Helicidae) populations from south-eastern and eastern parts of the modern area, Russ. J. Genetics: Appl. Res., 2016, vol. 6, no. 2, pp. 152-162.

Snegin, E.A. and Prisnyi, A.V., New data on the terrestrial mollusks of the Central Russian Upland, Nauch. Vedomosti Belgorod. Natl. Res. Univ., Ser. Estestv. Nauk, 2008, vol. 6, no. 3 (43), pp. 101-105. http://dspace.bsu.edu.ru/handle/123456789/15519.

Van de Peer, Y. and de Wachter, Y., TREECON for Windows: a software package for the construction and drawing of evolutionary trees for the Microsoft Windows environment, Comput. Appl. Biosci., 1994, vol. 10, no. 5 , pp. 69-70.

Voroshilova, I.S., Genetic markers in studies of dreissenides invasions (Dreissenidae, Bivalvia), Povolzhskii Ekol. Zh., 2015, no. 1, pp. 3-14.

Wright, S., Breeding structure of populations in relation to speciation, Am. Nat., 1940, vol. 74, no. 752, pp. 232-248.

Wright, S., Isolation by distance, Genetics, 1943, vol. 28, pp. 114-138.

Wright, S., Random drift and shifting balance theory of evolution, in Mathematical Topics in Population Genetics, Kojima, K.-I., Ed., Berlin: Springer-Verlag, 1970, pp. $1-31$.

Yeh, P.J. and Trevor, D.P., Adaptive phenotypic plasticity and the successful colonization of a novel environment, Am. Nat., 2004, vol. 164, pp. 531-542.

Translated by S. Semenova 